\title{
Die MCI im Wandel: User Experience als die zentrale Herausforderung?
}

\author{
Hans-Christian Jetter \\ Universität Konstanz, Arbeitsgruppe Mensch-Computer Interaktion
}

\section{Zusammenfassung}

Die Informationstechnologie hat heute einen Reifegrad erlangt, der stark umkämpfte Massenmärkte von interaktiven Produkten hervorgebracht hat. In diesen sind - wie auch in den herkömmlichen Konsumgütermärkten - nicht nur die pragmatische Produktqualität, sondern auch das Benutzungserlebnis und die Gesamtwirkung auf den Käufer entscheidend für den Markterfolg. Eine erfolgreiche Gestaltung dieser „User Experience“ geht dabei über die reine Softwareergonomie hinaus und erfordert interdisziplinäre Anstrengungen, die vom Design bis zur strategischen Unternehmenskommunikation reichen. Dieser Beitrag fasst die Sichtweisen zu „User Experience“ in der Literatur zusammen und argumentiert für eine Öffnung der Disziplin MCI für derartige Fragestellungen jenseits der Usability.

\section{$1 \quad$ Einleitung}

Ausgangspunkt dieser Betrachtung ist die Aussage von Patrick W. Jordan (ehemaliger „Head of Human Factors“ bei Philips Electronics) nach der Design eines der wenigen Gebiete ist, in denen Produkte heute überhaupt noch signifikante Marktvorteile erreichen können (Jordan 2000, 2ff.). Gerade in den umkämpften Massenmärkten interaktiver Produkte begnügen sich viele Hersteller nicht mehr damit, Funktionalität prinzipiell bereitzustellen, sondern erheben den Anspruch, dabei eine hochwertige und sympathische Erscheinung, einfache und effiziente Bedienung und große Benutzerzufriedenheit bei der Benutzung zu gewährleisten. Egal ob in Don Normans „Emotional Design“ (Norman 2004), in Karen Donoghues „Built for Use“ (Donoghue 2002), in Jesse J. Garretts „Elements of User Experience“ (Garrett 2002), bei der Gestaltung von Patrick W. Jordans „Pleasurable Products" (Jordan 2000) oder der pragmatischen und hedonischen Qualität 
im Sinne von Burmester et al. (2002): der Konsens lautet, dass für den Erfolg von Produkten ${ }^{1}$ am Massenmarkt eine individuell am Benutzer und seinen Werten ausgerichtete Gestaltung des Benutzungserlebnisses notwendig ist.

An sich ist eine derartige benutzer-zentrierte Denkweise innerhalb der Disziplin der Mensch-Computer Interaktion (MCI) schon lange selbstverständlich. Neu ist dabei aber auf welchen Ebenen die benutzer-orientierte Gestaltung für Produkte von Apple iPod bis Blackberry gefordert wird. Das positive Benutzungserlebnis (im allgemeinen als „User Experience“ oder „UX“ bezeichnet) umfasst dabei nicht nur die zweckrationale, effektive und effiziente Erreichung von Benutzerzielen, sondern behandelt auch eine Vielzahl anderer Qualitäten, wie z.B. Ästhetik (Tractinsky \& Hassenzahl 2005), Motivation (Hassenzahl 2003a), Credibility (Fogg \& Tseng 1999), Pleasure (Sengers 2003) oder kommunizierte Identität und Branding (Altobelli \& Sander 2001). Sie versucht dabei ganz im Sinne der von B. Joseph Pine II und James H. Gilmore (1999) propagierten „Experience Economy“ (Erlebnisökonomie), die zweckrationale Zielerreichung mit dem Produkt in eine ansprechende, motivierende, sympathische und - in jeder weiteren Beziehung - ,positive“ Erfahrung zu verwandeln. Eine gezielte Gestaltung und Evaluation dieser „User Experience“ geht demnach über das klassische Usability Engineering hinaus und adressiert sowohl rationale als auch irrationale Benutzerbedürfnisse, um die gewünschten Benutzerzahlen und Absatzziele zu erreichen. Es stellt sich daher die Frage, ob die MCI als Disziplin in Zukunft die Herausforderung einer benutzer-zentrierten Gestaltung von Produkten jenseits der Usability annehmen kann und möchte.

Dieser Beitrag soll zunächst eine Bestandsaufnahme zum Thema „User Experience“ leisten, um die stark divergierenden Konzepte aus Literatur und Praxis zusammenzufassen. Anschließend wird im Sinne eines Positionspapiers für eine Erweiterung der MCI auf der Basis ihrer eigenen Geschichte argumentiert. Mit einem Ausblick auf das Potential einer derartig erweiterten MCI schließt dieser Beitrag, der dabei als grundlegende Vorüberlegung zu verstehen ist, um Perspektiven für die zukünftige Forschungsarbeit und Möglichkeiten neuer interdisziplinärer Synergien aufzuzeigen.

\footnotetext{
${ }^{1}$ Als Produkt soll hier entsprechend Kuniavsky (2005) jede Schnittstelle zwischen einer Organisation und dem Benutzer oder Kunden verstanden werden, die in Form eines Geräts, einer Dienstleistung, eines Systems, einer Software oder einer Kombination aus allem angeboten wird. Dabei bezieht sich dieser Beitrag insbesondere auf Standardsoftware, E-Commerce Angebote, Kommunikationsdienste und (mobile) Information Appliances für den alltäglichen Gebrauch.
} 


\section{Was ist „User Experience“?}

Schon in den 1970er Jahren wurde in Veröffentlichungen der Informatik „User Experience“ als Bezeichnung für die praktischen Erfahrungen und Erlebnisse bei der Benutzung eines interaktiven Systems verwendet. Die Prägung von „User Experience“ als eigenständigen Begriff wird aber im Allgemeinen Don Norman zugeschrieben, der bei seiner Tätigkeit für Apple darunter die Gesamtwirkung aller bei der Benutzung verwendeten Gegenstände auf den Benutzer verstanden hat - angefangen von der Produktverpackung über die Gestaltung der Interaktion bis zum Grafikdesign auf dem Bildschirm (Norman 2004). Auch in weiterer Literatur lässt sich dieses Verständnis von „User Experience“ bis zu Apple zurückverfolgen, z.B. bei Russell (1998), der die dort seit 1995 existierende interdisziplinäre „User Experience Research Group" thematisiert. Daraufhin formierte sich eine bis heute anwachsende Zahl von interdisziplinären „User Experience“ Abteilungen in verschiedensten Organisationen, z.B. bei IBM, Xerox PARC, Microsoft, Nokia oder SAP. In der e-Commerce-Literatur wurde UX besonders in den Veröffentlichungen von Garrett (2002), Donoghue (2002) oder Kuniavsky (2003) thematisiert. Dass sie dabei mehr als nur ein marktwirksames dot-com-Synonym für Usability ist, zeigt sich darin, dass sich die renommierte British HCI Group Annual Conference 2005 unter dem Motto „The Bigger Picture“ dem Thema sogar in einem Keynote-Vortrag des „Head of Brand Experience“ vom Mobilfunkanbieter Orange widmete. Auch in der kommenden Neuauflage des Human-Computer Interaction Handbooks (Jacko \& Sears 2002) wird ein Kapitel speziell über „HCI and User Experience" erscheinen (Kuniavsky 2006). Weiterhin gilt der Begriff als zentraler Bestandteil der bald einsetzenden Werbekampagne für Microsofts neues Windows „Vista“, wodurch UX weltweit bei Nicht-Fachleuten popularisiert werden wird, so dass eine wissenschaftliche Bewertung dieses durch die Industrie geprägten Begriffs durch die MCI überfällig erscheint.

Einigkeit über die Übersetzung des Begriffs ins Deutsche (z.B. als „Benutzungserfahrung“ oder besser: „Benutzungserlebnis“) herrscht bislang nicht, weshalb der englische Begriff „User Experience“ im Rahmen dieses Beitrags beibehalten wird. Ebenso gibt es bisher keinen Konsens über eine präzise wissenschaftliche Definition und Abgrenzung dieses Begriffs, die bislang je nach Perspektive des Definierenden sehr unterschiedlich ausfallen.

Betrachtet man die Verwendung aus dem Umfeld der MCI oder Psychologie, z.B. im Kontext der „Funology“ oder des „Joy-of-Use“, herrscht Einigkeit 
darüber, dass die UX nicht nur durch die Effektivität, Effizienz und Zufriedenheit bei der Benutzung von Anwendungen geprägt ist (siehe Definition von Usability bzw. Gebrauchstauglichkeit in der ISO-Norm 924111), sondern ein ganzheitlicher Begriff von der Interaktion zwischen Mensch und Computer herangezogen wird, der auch die Motivation, Identifikation, Ästhetik oder - in der Sprache mancher Käuferschichten - die „Coolness“" oder "Sexiness" umfasst. Somit beinhaltet sie auch die Wirkung von Faktoren, die innerhalb der MCI bisher nur von wenigen (z.B. Tractinsky, Fogg, Hassenzahl etc.) individuell betrachtet wurden und im Allgemeinen als Grenzbereiche der MCI eher undifferenziert unter „Zufriedenheit" eingeordnet wurden. Nichtsdestotrotz gelten diese hedonischen und emotionalen Qualitäten z.B. nach der Meinung von Jordan (2000, 12ff.) oder Norman (2004) als entscheidend für die Frage, ob ein Benutzer ein Produkt über seine pragmatische Qualität hinaus als ,„pleasurable“ empfindet. Nach Hassenzahl (2003b) muss ein erfolgreiches Produkt beim Käufer die kognitive Konstruktion eines Charakters auslösen, der neue Eindrücke, Möglichkeiten und Einsichten verspricht (Stimulation), der den Besitzer nach außen hin auf die gewünschte Art repräsentiert (Identifikation) und positive Erinnerungen und Assoziationen auslöst (Evokation).

Betrachtet man die Verwendung im Kontext des e-Commerce (z.B. durch Garrett, Donoghue oder Kuniavsky) kommt innerhalb der User Experience auch dem wirtschaftlichen oder organisationalen Kontext eine besondere Rolle zu. Die User Experience beinhaltet daher nicht nur das subjektive Erlebnis des Benutzers, sondern auch inwieweit dieses mit den strategischen Kommunikations- und Businesszielen übereinstimmt, die die Organisation hinter dem Produkt verfolgt. Hier werden insbesondere Marketing und „Internet-Branding“ relevant (Altobelli \& Sander 2001). Nach Donoghue ist dabei entscheidend, dass die Marke (oder „Brand“) während der Benutzung ihr Markenversprechen, also die Erwartungshaltung, die ein Kunde gegenüber einer Organisation und ihren Produkten aufgebaut hat, möglichst effektiv und angemessen einlöst. Anforderungen an die User Experience werden dementsprechend nicht nur durch den Usability Engineer (stellvertretend für den Benutzer) formuliert, sondern sind auch Bestandteile der übergeordneten strategischen Unternehmenskommunikation.

Nach Garrett und Donoghue versucht die „User Experience“ dabei insbesondere ein Gleichgewicht zwischen Benutzerzielen und Zielen der Organisation zu schaffen. Kuniavsky grenzt die Gestaltung der UX deswegen von der Usability-fokussierten MCI ab, die implizit davon ausgeht, dass es das 
alleinige Ziel der Gestaltung sei, hohe Gebrauchstauglichkeit für den Endnutzer anzubieten. Kuniavsky $(2006,5)$ zeigt dabei auf, wie andere Werte stattdessen mit ihr in direkten Konflikt treten können und dabei in der Praxis auch Usability-Ziele bewusst überlagern.

Abbildung 1 fasst das Verhältnis zwischen User Experience, MCI und Usability vereinfacht zusammen, um hier als Gebrauchsdefinition für die weitere Diskussion bereitgestellt zu werden.

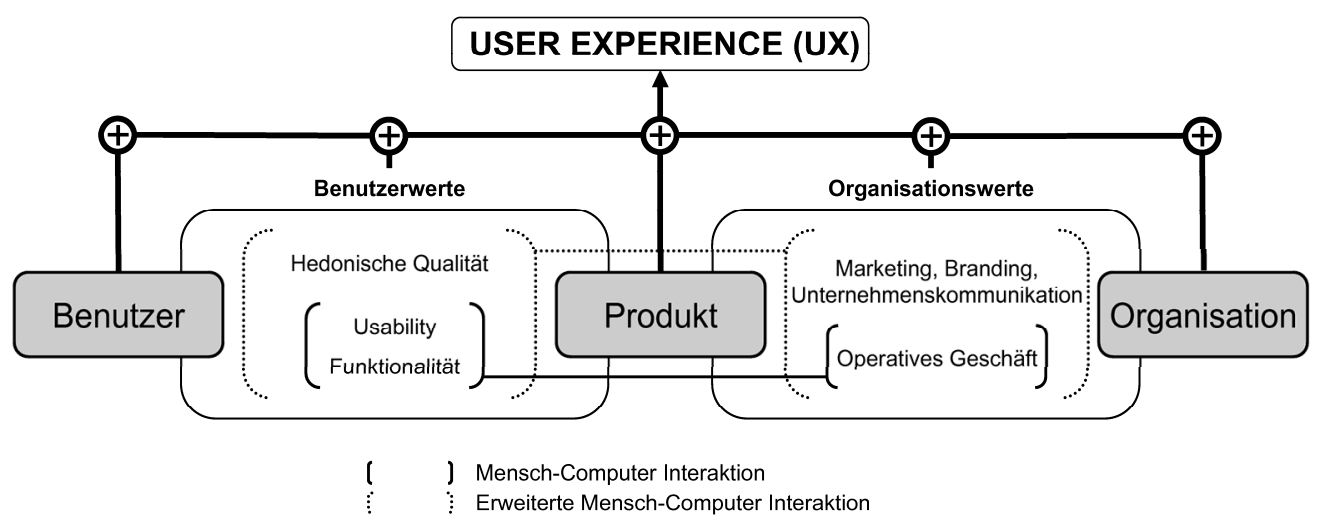

Abbildung 1 - Elemente der User Experience (UX), sowie die Ziele und Qualitätsfaktoren der heutigen MCI und die einer ,,erweiterten MCI" mit dem Fokus auf User Experience

\section{User Experience und Usability}

Betrachtet man die Entwicklung von der Industrie- zur Informationsgesellschaft, so ist der wachsende Einfluss nichtrationaler Bedürfnisse nicht zu leugnen. Nach dem Soziologen und Arbeitswissenschaftler Böhle (2003) galt die Abgrenzung von Arbeit gegenüber anderen Lebensbereichen und Tätigkeiten als ein Grundpfeiler industrieller Gesellschaften. Mit dem modernen Rationalismus wurde dabei das „Andere der Vernunft" überhaupt erst als eigenständiger Bereich geschaffen, wobei eine „Abwertung des Nichtgeplanten, Nichtzweckgerichteten und Nichtrationalen" stattgefunden hat. Dies erscheint gerade in der Umgebung eines erstarkenden Taylorismus als wirtschaftlich sinnvoll. In einer „New Economy“ gibt es jedoch neuartige Möglichkeiten der ganzheitlichen Befriedigung rationaler und nichtrationaler Bedürfnisse - sei es aufgrund der Vielzahl von individuellen Produkten oder völlig neuen gestalterischen Möglichkeiten oder Marktmechanismen. 
In Böhles Augen ist das „,beharrliche Festhalten an der Vorherrschaft der Ratio" heute nicht mehr geeignet, sich der ankündigenden Herausforderungen in Praxis und Wissenschaft anzunehmen. Derartige Standpunkte sind auch von anderen aufgegriffen worden, z.B. von Jordan $(2000,7)$, der als Vertreter von „Pleasurable Products“ seinen Standpunkt zu diesem „beharrlichen Festhalten“ wie folgt formuliert: „Usability-based approaches [...] encourage a limited view of the person using the product. This is - by implication if not by intention - dehumanising."

Auch eine Betrachtung der bisherigen Geschichte der MCI kann als Argument für eine Erweiterung der Disziplin über die reine Usability hinaus herangezogen werden: Obwohl komplexe Eigenschaften wie „Benutzerfreundlichkeit" zunächst schwer definierbar und kaum Ansatzpunkte für ihre gezielte Gestaltung vorhanden waren, konnte die wissenschaftliche Auseinandersetzung damit kreative Vorreiter der MCI zu revolutionären Designs inspirieren - im Falle von Maus, GUI oder Hypertext sogar bereits Jahrzehnte vor deren Einführung in die Massenmärkte (Myers 1998).

In der darauf folgenden zweiten Phase der MCI wurden daher zunehmend Experten für die Gestaltung von Benutzungsschnittstellen herangezogen, dies aber oft nur in den Endphasen einer Entwicklung. Der Gestaltungsspielraum und der Mehrwert für den Benutzer war daher sehr begrenzt (Jordan 2000, 2ff.). Erfolgreich waren jedoch die Bemühungen mit dem Begriff der Usability oder Gebrauchstauglichkeit ein allgemein anerkanntes und differenziertes Modell von „Benutzerfreundlichkeit“" in der ISO $9241 \mathrm{zu}$ etablieren.

Als ,dritte Phase“ bezeichnet Jordan die heutige Situation, in der die meisten großen Hersteller, die Berücksichtigung von „Human Factors“ in ihren Entwicklungsprozessen mehr oder weniger systematisch verankert haben, um damit die Usability in eine kontrollierbare Qualität und in einen Wettbewerbsvorteil innerhalb der wachsenden Massenmärkte zu verwandeln. In diesen stellen sich aber zunehmend neue Herausforderungen: Die Industrie sieht sich mit ungeheurer Popularität von interaktiven Produkten und einer Vielzahl von Wettbewerbern konfrontiert, deren Angebote sich oftmals in ihrer Funktionalität nur marginal unterscheiden. Derzeit prominentes Beispiel dürfte der iPod von Apple sein, der letztlich spät in einen Markt von sehr ähnlichen MP3-Playern eingedrungen ist, obwohl er bei der Markteinführung als technologisch nicht überlegen und preislich als unattraktiv empfunden wurde. Aus der rein rationalen Perspektive der Usability (siehe z.B. Kritik an der Usability des Apple iPod von Dougherty (2004)) war der iPod kein 
überlegenes Produkt, bot aber durch das koordinierte Zusammenspiel von Design, Benutzungsschnittstelle und dem Webangebot iTunes eine überlegene User Experience, die das Brand „Apple“ weiter verfestigte. Letztlich ist der iPod mit einem Marktanteil von über 90\% (laut Apple) in den USA zu einem Synonym für mobile MP3-Player geworden und hat mit dem Begriff „Podcasting“ sogar die Bezeichnung für ein viel diskutiertes Massenmedium im Netz geprägt.

Prinzipiell stehen die Hersteller von Informationstechnologie also wieder vor einem Problem aus der Anfangszeit der MCI und zwar vor dem der Bändigung schwer greifbarer Begriffe und Anforderungen (damals „Benutzerfreundlichkeit", heute „User Experience“). Die Frage ist, ob und wie User Experience innerhalb eines kontrollierten Entwicklungsprozesses für interaktive Produkte differenziert betrachtet und gewährleistet werden kann. Dabei sind vonseiten der MCI zwei Reaktionen denkbar:

Definiert man die MCI als eine Disziplin der Gestaltung und Evaluation gebrauchstauglicher Produkte im Sinne der ISO-Norm, reduziert sich ihr Beitrag zur Gestaltung der UX entsprechend auf die pure Usability. Falls notwendig, ließen sich dabei viele der bisher schwer quantifizierbaren hedonischen oder emotionalen Einflussgrößen als Beeinträchtigung der Dimension „Zufriedenheit“ im Sinne der ISO 9241-11 behandeln. Die MCI erhebt dabei bewusst nicht den Anspruch, eine ganzheitliche Design-Disziplin für UX zu werden, sondern fokussiert sich auf die Rolle einer ,problem-solving discipline“" (Jordan 2000, 4) zur Vermeidung schlechter Usability.

Eine andere Sicht der Dinge wäre nach der Definition der MCI im ACM SIGCHI Curriculum möglich: „Human-computer interaction is a discipline concerned with the design, evaluation and implementation of interactive computing systems for human use and with the study of major phenomena surrounding them." Das Beispiel des iPods belegt, wie stark der Einfluss von hedonischen Qualitäten auf die Toleranz gegenüber mangelnder Usability oder geringerer Funktionalität ist. Dieses bedeutende Phänomen kann demnach also auch als ein Thema für die MCI betrachtet werden.

Schützenhilfe erhält dieser Standpunkt unter anderem von Gilbert Cocktons „value-centred HCI“ (Cockton 2004), die das Wertesystem des Benutzers und der weiteren Stakeholder in das Zentrum der MCI stellt. Da sich dieses Wertesystem für jeden Benutzer unterscheidet und ständigen gesellschaftlichen Veränderungen unterworfen ist, stellt Cockton eine pragmatische Wertschöpfung durch die MCI über die Gewinnung von vermeintlichen 
ergonomischen „Wahrheiten“. Er schreibt: „Traditional disciplines are about delivering truth. The goal of HCI is to deliver value." Um Produkte mit „Wert“ für den Benutzer (oder auch andere Stakeholder) zu schaffen, ist es notwendig, sich am jeweiligen Wertesystem des Benutzers, beteiligter Organisationen oder anderer Stakeholder zu orientieren. „A focus on value creates a paradoxical discipline that fuses subjectivity and objectivity in a single process. HCI must be objectively systematic and reliable in the pursuit of subjective value."

\section{$4 \quad$ Fazit}

Es stellt sich die grundsätzliche Frage nach der Aufgabe der MCI: soll sie sich als Disziplin der Ergonomie der Aufgabe widmen, das Ideal einer hypothetischen „optimalen“ Usability durch weiteren Ausbau von Theorien und Methoden zu verfolgen? Oder sieht sie ihre Aufgabe darin, die Hersteller von interaktiven Produkten bei der Erreichung eines pragmatischen Gleichgewichts zwischen ,,angemessener“ Usability, ,,angemessenem“ Benutzungserlebnis und Rentabilität des Entwicklungsprozesses zu unterstützen?

Sollten die Stakeholder eine attraktive Gestaltung oder die Erfüllung eines Markenversprechens als wesentlichen Wert bei der Benutzung betrachten, so sollte sich eine anwendungs-orientierte MCI davor sicher nicht verschließen. Mit einer Beschränkung auf die alleinige Optimierung der zweckrationalen Gebrauchstauglichkeit würde die MCI entscheidende Fragen der Benutzerakzeptanz ausblenden und diese anderen Disziplinen überlassen. Anstelle einer Überbetonung der „reinen Lehre“ der Ergonomie könnte die MCI stattdessen auch benachbarte Disziplinen bei der gezielten Gestaltung der User Experience unterstützen und als Vermittler zwischen strategischer Planung, Qualitätssicherung und gestalterischer Kreativität im Sinne von Hartwig und Hassenzahl (2005) fungieren. Dabei könnten die bisherigen Erfolge der MCI eine hervorragende Ausgangsbasis liefern:

1. Die MCI hat durchdachte Vorgehensmodelle geschaffen und vermittelt mit dem Usability Engineering erfolgreich zwischen Management, Entwicklern und Benutzern unter Berücksichtung der Kosten, der individuellen Interessen und der Rechte aller Stakeholder.

2. Die MCI beherrscht die Erfassung von Requirements im Dialog mit allen Stakeholdern und beherrscht die Erfassung und Modellierung 
von Benutzergruppen und deren Zielen, wodurch sich Möglichkeiten der Synergie mit dem Marketing und der Produktplanung ergeben.

3. Die MCI liefert Methoden und Werkzeuge zur Formulierung von Entwicklungszielen und zur deren praktischen Bereitstellung im Gestaltungsprozess für Interface Designer und Software Entwickler.

4. Die MCI besitzt eine umfangreiche Wissensbasis praxisrelevanter Erkenntnisse aus der Psychologie und den Kognitionswissenschaften, die als anwendungs-orientierte Heuristiken zum Einsatz kommen.

5. Die MCI besitzt Methoden zur systematischen Berücksichtigung von Requirements, Benutzermodellen und kognitiven Restriktionen während der Gestaltung von Produkten mit völlig neuen Designs oder mit bereits bewährten Designpatterns.

6. Die MCI hat Erfahrung in der Anwendung von verschiedensten Evaluationsmethoden, die sich durch neue Modelle von UX und durch verbesserte empirische Methodik ausweiten lassen könnten, um auch die Qualitätssicherung jenseits der Usability zu bereichern.

Sowohl für die MCI als wissenschaftliche Disziplin als auch für die Industrie würden sich mit einer erweiterten MCI neue Potentiale eröffnen:

Anstelle der Verhinderung von Negativeffekten durch schlechte Usability als „dissatisfier" hätte die MCI die Möglichkeit, auch proaktiv an der Gestaltung und Bewertung von „satisfiern“ (z.B. durch hochattraktives Interface- und Interaktionsdesign oder immersive Ein- und Ausgabe) mitzuwirken. Das Selbstverständnis als gestaltende ,positive“ MCI würde dabei durchaus in der Tradition der Pioniere dieser Disziplin stehen. Vor diesem Hintergrund wird es sich als lohnenswert erweisen, Berührungsängste mit schwer greifbaren Größen und Faktoren oder anderen Disziplinen abzulegen, um damit einen Schritt zu wagen, den letztendlich auch die Informatiker und Ingenieure der Vergangenheit bei der Begründung der MCI taten.

\section{$5 \quad$ Literatur}

Altobelli C. F.; Sander M. (2001): Internet-Branding. Marketing und Markenführung im Internet. Lucius \& Lucius.

Böhle, F.: Anders arbeiten - anders lernen. In: Personalführung, Heft 1, 2003, S. 1-3. 
Burmester, B.; Hassenzahl, M.; Koller, F. (2002): Usability ist nicht alles - Wege zu attraktiver Software. In: i-com. Zeitschrift für interaktive und kooperative Medien. Ausgabe 1/2002, München: Oldenbourg, S. 32-40.

Cockton, G. (2004): Value-centred HCI. Proceedings of the third Nordic conference on Humancomputer interaction, Tampere Finland. ACM Press.

Donoghue, K. (2002): Built for Use: Driving Profitability Through the User Experience. McGraw Hill.

Dougherty, B. (2004): iPod Usability Critique. http://www.unc.edu/ $\operatorname{bretd} / 222$ ipodusecritique.htm (Stand 15.02.2006)

Fogg, B. J.; Tseng, H. (1999): The elements of computer credibility. In: Proceedings of the SIGCHI Conference on Human Factors in Computing Systems: the CHI Is the Limit (Pittsburgh, Pennsylvania, United States, May 15 - 20, 1999). CHI '99. ACM Press, New York, NY, 80-87.

Garrett, J. J. (2002): The Elements of User Experience: User-Centered Design for the Web. New Riders Press.

Hartwig, R.; Hassenzahl, M. (2005): Certified Fun - Stehen hedonische Qualitätsaspekte und Qualitätssicherung im Widerspruch? In: Marc Hassenzahl, Matthias Peissner M. (Hrsg.), Usability Professionals 2005. Proceedings des dritten GC-UPA-Tracks. German Chapter der Usability Professionals' Association e.V., Stuttgart

Hassenzahl, M. (2003a): Attraktive Software - Was Gestalter von Computerspielen lernen können, In: Joachim Machate, Michael Burmester (Hrsg.), User Interface Tuning. Benutzungsschnittstellen menschlich gestalten, Frankfurt: Software \& Support Verlag.

Hassenzahl, M. (2003b): The Thing and I: Understanding the Relationship Between User and Product, In: Mark A. Blythe, Kees Overbeeke, Andrew F. Monk, Peter C. Wright (Hrsg.), Funology - From Usability to Enjoyment, Dordrecht: Kluwer Academic Publishers, S. 31-42.

Jacko, J. A.; Sears, A. (Hrsg.) (2003): The Human-Computer Interaction Handbook: Fundamentals, Evolving Technologies and Emerging Applications. Lawrence Erlbaum.

Jordan, P. W. (2000): Designing Pleasurable Products. London: Taylor \& Francis.

Kuniavsky, M. (2003): Observing the User Experience: A Practitioner's Guide to User Research. Morgan Kaufmann.

Kuniavsky, M. (2005): User Experience and HCI (Draft). URL: http://www.orangecone.com/hci_UX_chapter_0.7a.pdf (Stand 16.03.2006)

Myers, B. A. (1998): A Brief History of Human Computer Interaction Technology. ACM Interactions. Vol. 5, no. 2, March 1998. pp. 44-54.

Norman, D. A. (2004): Emotional Design. New York: Basic Books.

Pine B. J. II; Gilmore J. H. (1999): The Experience Economy: Work Is Theatre \& Every Business a Stage. Harvard Business School Press.

Russell, D. M. (1998): User experience research group: understanding the complete user interaction. In: ACM SIGCHI Bull. 30, 2 (Apr. 1998), 90-94.

Tractinsky, N.; Hassenzahl, M. (2005): Arguing for Aesthetics in Human-Computer Interaction, In: icom. Zeitschrift für interaktive und kooperative Medien. Vol. 4. Issue 3, München: Oldenbourg, S. 66-68. 